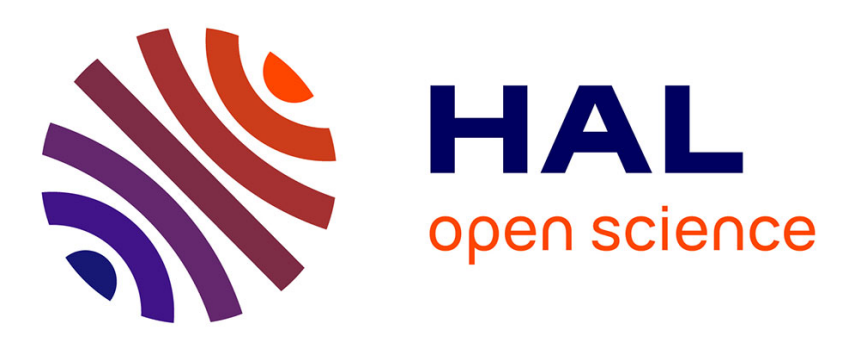

\title{
Sizing of a heterogeneous fleet of robots in a logistics warehouse
}

Achraf Rjeb, Jean-Philippe Lucien Gayon, Sylvie Norre

\section{To cite this version:}

Achraf Rjeb, Jean-Philippe Lucien Gayon, Sylvie Norre. Sizing of a heterogeneous fleet of robots in a logistics warehouse. 2021 IEEE 17th International Conference on Automation Science and Engineering (CASE), Aug 2021, Lyon, France. pp.95-100, 10.1109/CASE49439.2021.9551509 hal-03149661

\section{HAL Id: hal-03149661 https://hal.science/hal-03149661}

Submitted on 23 Feb 2021

HAL is a multi-disciplinary open access archive for the deposit and dissemination of scientific research documents, whether they are published or not. The documents may come from teaching and research institutions in France or abroad, or from public or private research centers.
L'archive ouverte pluridisciplinaire HAL, est destinée au dépôt et à la diffusion de documents scientifiques de niveau recherche, publiés ou non, émanant des établissements d'enseignement et de recherche français ou étrangers, des laboratoires publics ou privés. 


\title{
Fleet-sizing of robots in a logistics warehouse
}

Transport operation between reception area and storage area

\author{
A. RJEB ${ }^{*}$, J-P. GAYON ${ }^{* *}$, S. NORRE ${ }^{* * *}$ \\ LIMOS UMR CNRS 6158 - Université Clermont-Auvergne, \\ Campus Universitaire des Cézeaux, 63178 AUBIERE - FRANCE \\ *Achraf.RJEB@uca.fr \\ ** J-Philippe.GAYON@uca.fr \\ *** Sylvie.NORRE@uca.fr
}

\begin{abstract}
We are interested in the problem of determining the size of a fleet of robots able to perform transport operations in a logistics warehouse. The operations are divided into several phases: loading, loaded travel, unloading and empty travel. We first determine the minimum number of robots needed to transport a set of homogeneous loads from point A to point B over a determined time interval. For the same number of robots, many assignments exist. We determine the assignment that minimizes the makespan, which is also the one which balances the loads between robots. If we consider an infinity of pickup stations, we obtain a simple analytical expression to find the optimal number of robots. With a single pickup station, we present a simple algorithm that computes the optimal fleet size. Finally, we show that the extension with heterogeneous loads can be formulated as a bin packing problem.
\end{abstract}

Keywords: Fleet-sizing, automated warehouse, internal logistics, transport operations, robots.

\section{INTRODUCTION}

The concept of multi-robot system (MRS) appeared in 1989 in order to replace a large robot by a set of small robots (Mouad, 2014). For multi-robot systems in the context of logistics warehouses, the literature has since been enriched by focusing in particular on the following fields of study: guide-path design, fleet-sizing, vehicle scheduling, positioning, battery management, deadlock management.

One of the major problems of logistics warehouses is the problem of fleet-sizing which consists in determining the optimal number of vehicles able to perform all requested tasks in a given time interval with a minimum total cost (Sinriech et al., 1992). Indeed, the number of vehicles greatly influences multi-robot systems performance and determining the right type and number of vehicles is essential.

The fleet-sizing problem has two aspects: an operational aspect and an economic aspect (Sinriech et al., 1992). For the operational aspect, (Egbelu, 1987) presents six strategic factors that influence strongly the estimation of the number of vehicles: (1) The system layout, (2) the location of load transfer points, (3) trip exchanges between workcenters per unit time, (4) vehicle-dispatching strategy, (5) system reliability, (6) travel speed.

For the economic aspect, the total costs of robots can be divided as follows (Sinriech et al., 1992):

\footnotetext{
* Thesis co-financed by the European Union within the framework of the European Regional Development Fund ERDF
}

- Operating costs including everything that is related to the operations such as maintenance costs, energy costs, etc.

- Design costs including the software costs (scheduling, routing, dispatching, etc) and the hardware costs (batteries / chargers, communication links, etc).

- Guide-path design cost related to the the guide-path configuration.

The literature considers the fleet-sizing problem in different ways (see e.g. (Choobineh et al., 2012) and (Ganesharajah et al., 1998) for reviews).

According to (Choobineh et al., 2012), the sizing models presented in the literature can be classified into four categories of approaches: (1) discrete and continuous event simulation models, (2) calculus approaches including a set of simple computations, (3) deterministic operations research models including linear and integer programming, (4) stochastic models including queuing theory.

Since the first research works dealing with this fleet-sizing problem, the analytical models used to determine the optimal size of the fleet are not entirely reliable in terms of optimization. Indeed, analytical models today are only used to find an initial number of robots which is not necessarily optimal before continuing with the sizing procedure. In order to refine the analytical results, most of the work is based on the number of robots found analytically to launch an optimization study through algorithmic or simulation models. 
Among the most used models to start the sizing procedure, we can cite (Egbelu, 1987) model. This model calculates the number of robots in the fleet according to the following expression:

$$
m^{*}=\frac{\text { total operating time }}{\text { time interval }}
$$

Although this model takes into account several constraints (empty circulation of vehicles, number of pickup / delivery stations per zone ...), it does not take the integer values into consideration which strongly impacts the optimality of the solution.

Recently, (Vivaldini et al., 2016) improved this model by taking into account integer values. On the other hand, this model remains limited from an optimization point of view since it does not take into account the waiting times caused by several problems (unavailability of the load to be transported, loading station occupied, etc). Indeed, (Vivaldini et al., 2016) consider the number found by this model as a starting point for a second optimization step.

The model of (Choobineh et al., 2012) has been used by several researchers to find an initial value for the size of the fleet. This model was used recently by (Chawla et al., 2018b) and (Chawla et al., 2019) in order to show that the values found analytically can be optimized through optimization tools like (gray wolf optimization algorithm, particle swarm algorithm).

\section{TRANSPORT OF HOMOGENEOUS LOADS}

In this section, we present a transport model for loads with the same type.

\subsection{Notations and assumptions}

Fig. 1 illustrates an homogeneous fleet of robots that transport homogeneous loads from zone $A$ (reception) to zone $B$ (storage). A robot moves loaded from $A$ to $B$ before crossing the same empty distance from $B$ to $A$.

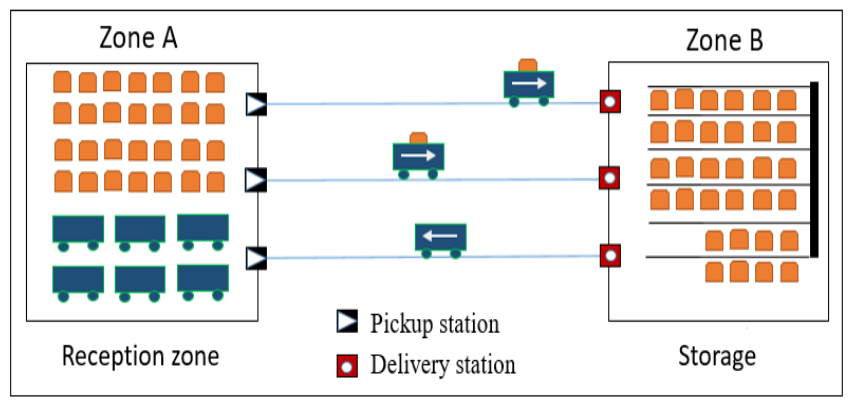

Fig. 1. Transport model between A and B

\section{Notations}

$-t_{l}$ : loading time

$-t_{u}$ : unloading time

$-T$ : time limit

- $d_{A B}$ : distance between $A$ and $B$

- $n$ : number of loads to transport from $A$ to $B$ during $T$

- $m$ : number of robots
$-n_{A}$ : number of pickup stations in $A$

$-n_{B}$ : number of delivery stations in $B$

$-v_{l}$ : loaded robot travel speed (uniform)

$-v_{e}$ : empty robot travel speed (uniform)

\section{Assumptions}

- The robot parking is located at point $A$

- Transport is unitary: each robot carries at most a single load at a given time

- The loads to be transported are always available

- Deadlock during travel and idleness of robots are not taken into account

- The battery charging problem is not taken into account

Objective function The purpose of this study is to determine the minimum number of robots $m^{*}$ allowing to transport the $n$ loads in the time interval $[0, T]$.

Many load assignments can exist for the same number of robots. We will denote by $C_{j}$ the time at which robot $R_{j}$ has terminated its last transport cycle. We will also denote by $C_{\max }=\max _{j} C_{j}$ the makespan which represents the time at which all loads have been transported and all robots are back in $A$. We will focus in particular on the assignment which minimizes the makespan but also on the one which distributes the loads in the most balanced way between robots.

Cycle time of a robot We will denote by $p$ the cycle time of a robot for one load (loading, loaded travel, unloading and empty travel):

$$
p=d_{A B} \cdot\left(\frac{1}{v_{l}}+\frac{1}{v_{e}}\right)+t_{l}+t_{u}
$$

\subsection{Many pickup / delivery stations}

We assume in this part that there are at least as many pickup / delivery stations as there are robots at the optimum $\left(n_{A} \geq m^{*}\right.$ and $\left.n_{B} \geq m^{*}\right)$. Under this assumption, there is no waiting time for loading or unloading.

We can notice that there is a solution to the problem if and only if the cycle time $p$ is less than or equal to the time interval $[0, T]$. We will assume in the rest of this paper that $p \leq T$ in order to place ourselves in the case where a solution exists.

Optimal number of robots The maximum number of loads $n^{\max }$ transported by a robot on the time interval $[0, T]$ must satisfy the inequality $\left(n^{\max } p \leq T\right)$. The number $n^{\max }$ must be integer, which implies that

$$
n^{\max }=\left\lfloor\frac{T}{p}\right\rfloor .
$$

where $\lfloor x\rfloor$ is the floor function of $x$. We will also denote by $\lceil x\rceil$ the ceiling function of $x$.

The maximum number of loads transported by $m$ robots, denoted by $N_{\max }(m)$, is then

$$
N_{\text {max }}(m)=m\left\lfloor\frac{T}{p}\right\rfloor .
$$

In order to be able to transport the set of $n$ loads, it is necessary that $N_{\max }(m) \geq n$, so 


$$
m \geq \frac{n}{\left\lfloor\frac{T}{p}\right\rfloor} .
$$

The optimal number of robots is the smallest value of $m$ which satisfies (1), so we have

$$
m^{*}=\left\lceil\frac{n}{\left\lfloor\frac{T}{p}\right\rfloor}\right\rceil \text {. }
$$

Robots load balancing There can be several ways of assigning loads between the $m^{*}$ robots. The most balanced solution is the one that assigns the load with the objective of balancing the use of robots as much as possible. More precisely, it is the solution which minimizes the maximum difference between the different end dates $\max _{i, j}\left|C_{i}-C_{j}\right|$.

If $\frac{n}{m^{*}}$ is an integer, then it is possible to distribute the loads in a perfectly balanced way between the robots by assigning $\frac{n}{m^{*}}$ loads to each one.

If $\frac{n}{m^{*}}$ is not an integer, the robots will transport, in the most balanced solution, either $\left\lfloor\frac{n}{m^{*}}\right\rfloor$ loads, or $\left\lceil\frac{n}{m^{*}}\right\rceil=$ $\left\lfloor\frac{n}{m^{*}}\right\rfloor+1$ loads.

Let $s$ be the number of robots carrying $\left\lfloor\frac{n}{m^{*}}\right\rfloor$ loads. Thus we have $\left(m^{*}-s\right)$ other robots carrying $\left\lceil\frac{n}{m^{*}}\right\rceil=\left\lfloor\frac{n}{m^{*}}\right\rfloor+1$ loads. The total number of loads to be transported being $n$, we have

$$
n=s\left\lfloor\frac{n}{m^{*}}\right\rfloor+\left(m^{*}-s\right)\left(\left\lfloor\frac{n}{m^{*}}\right\rfloor+1\right) .
$$

which implies that

$$
s=\left\lceil\frac{n}{m^{*}}\right\rceil m^{*}-n .
$$

We notice that if $\frac{n}{m^{*}}$ is an integer, this last formula remains correct by giving $s=0$.

In the most balanced solution, the end date of the latest robot finishing its cycle is $C_{\max }$ where

$$
C_{\max }=\left\lceil\frac{n}{m^{*}}\right\rceil p
$$

We notice that the most balanced solution is also the one which minimizes the $C_{\max }$.

Let us now summarize our main results in the following theorem.

Theorem 1. When considering an infinity of pickup stations, the optimal number of robots is

$$
m^{*}=\left\lceil\frac{n}{\left\lfloor\frac{T}{p}\right\rfloor}\right\rceil \text {. }
$$

In the most balanced solution, noting $s=\left\lceil\frac{n}{m^{*}}\right\rceil m^{*}-n$, we have

- $s$ robots carrying $\left\lfloor\frac{n}{m^{*}}\right\rfloor$ loads

- $\left(m^{*}-s\right)$ robots carrying $\left\lceil\frac{n}{m^{*}}\right\rceil$ loads.

Finally, the most balanced solution is also the one that minimizes the makespan where

$$
C_{\max }=\left\lceil\frac{n}{m^{*}}\right\rceil p .
$$

Figs. 2 and Fig. 3 present two solutions for a transport model with an infinity of pickup stations and an optimal number of robots $m^{*}=4$. In the most balanced optimal solution (Fig. 2), 1 robot carries 4 loads and 3 robots carry 3 loads. In the unbalanced solution (Fig. 3), 3 robots carry 4 loads and 1 robot carries 1 load.

We can notice that the two solutions have the same makespan $C_{\max }=28$. On the other hand, the most balanced solution minimizes the differences between all the $C_{j}$ which guarantees the balanced use of robots for this model.

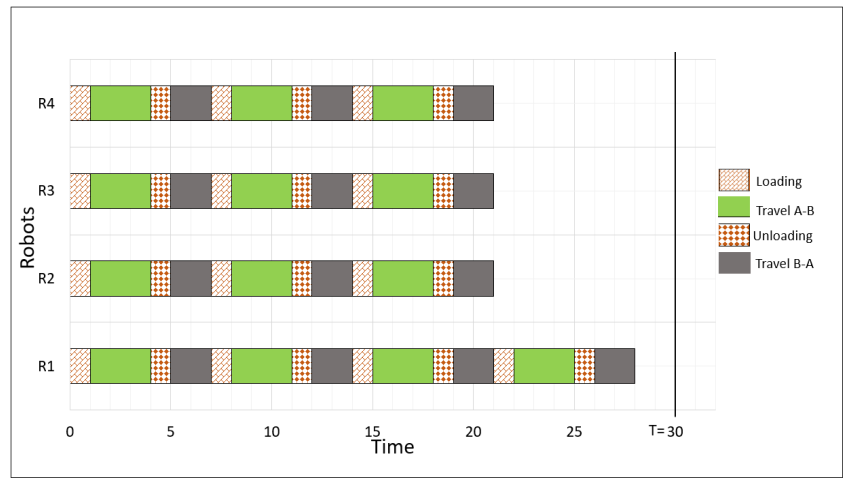

Fig. 2. Balanced solution

$\left(n=13, t_{l}=1, p=7, T=30, m^{*}=4\right)$

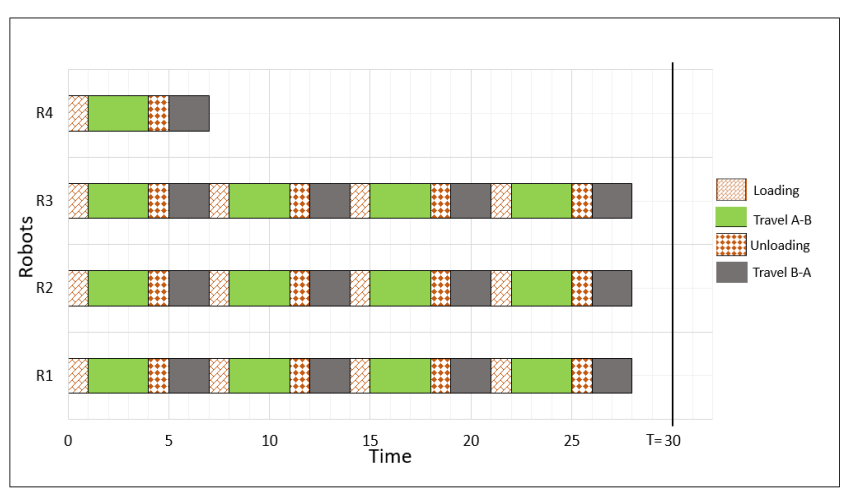

Fig. 3. Unbalanced solution

$\left(n=13, t_{l}=1, p=7, T=30, m^{*}=4\right)$

Continuous approximation If we allow a non-integer number of robots and loads, the optimal number of robots can be simplified to $m^{*}=n p / T$ and all the robots carry $T / p$ loads with a makespan $C_{\max }=T$. These results are consistent with (Egbelu, 1987).

This approximation is good if $p$ is much shorter than $T$ and the optimal number of robots is large, i.e. if $n p / T$ is large.

\subsection{Single pickup station}

We assume in this section that the system contains a single pickup station at point $A$ and at least one delivery station at point $B$, thus, $n_{A}=1$ and $n_{B} \geq 1$.

With a single pickup station, only one robot can load at a given time. We will denote by $R_{j}$ the robot starting in 
$j$-th position with $\left(1 \leq j \leq m^{*}\right)$. During the first load, $R_{1}$ does not wait, $R_{2}$ waits $t_{l}$ and more generally $R_{j}$ waits $(j-1) t_{l}$. For the following loadings, we must distinguish three cases:

- Case 1 : Waiting for first loading only $\left(p \geq m \cdot t_{l}\right)$

In this situation, when $R_{1}$ returns for the first time to $A$, robots $R_{2}, \cdots, R_{m}$ have completed their first loading. In this case, $R_{1}$ never waits to load and the others wait only for their first loading (see example in Fig. 4).

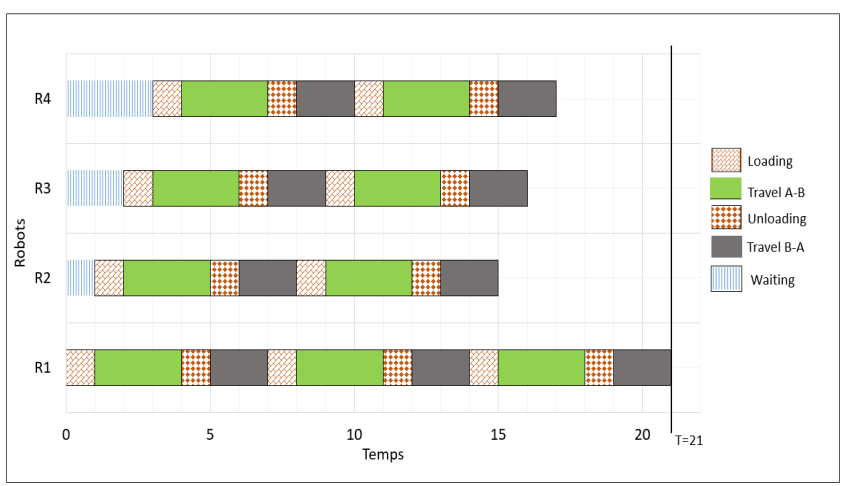

Fig. 4. Waiting for first loading only $\left(p \geq m \cdot t_{l}\right)$

$$
\left(n=9, t_{l}=1, p=7, T=21, m^{*}=4\right)
$$

- Case 2 : Waiting for every loading $(m-1) t_{l}<p<m t_{l}$

In this situation, when $R_{1}$ returns for the first time to $\mathrm{A}, R_{m}$ is being loaded. Note that the robots wait for $\left(m \cdot t_{l}-p\right)$ from their second loading. We observe for example in Fig. 5 that $R_{1}$ is forced to wait, on its return to $A$, for the end of loading of $R_{5}$.

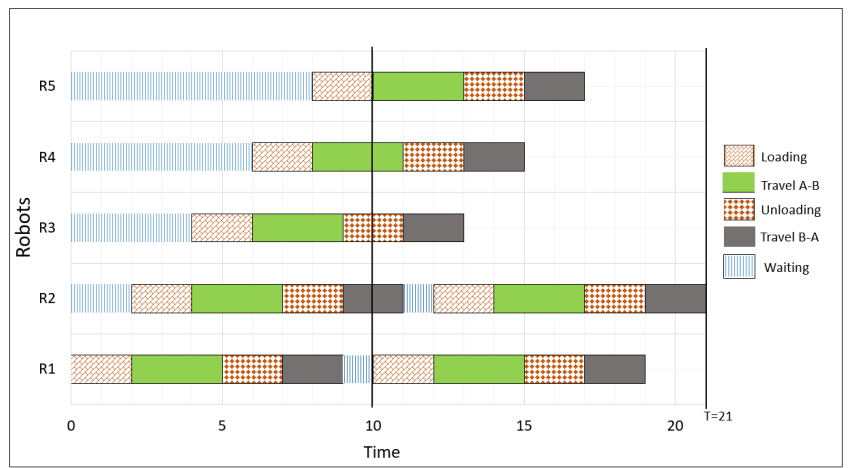

Fig. 5. Waiting for every loading $(m-1) \cdot t_{l}<p<m \cdot t_{l}$ $\left(n=7, t_{l}=2, p=9, T=21, m^{*}=5\right)$

- Case 3: Unnecessary robots $p \leq(m-1) \cdot t_{l}$

Here, when $R_{1}$ returns for the first time in $A, R_{m}$ has not yet started its first loading. Hence, there is at least one useless robot. We will therefore not consider this case subsequently.

Optimal number of robots We denote by $n_{j}^{\max }$ the maximum number of loads transported by $R_{j}$. In order to find $n_{j}^{\max }$, we will distinguish the two first cases identified previously.
- Case 1: The number of robots $m$ is integer, so, the condition $m \cdot t_{l} \leq p$ is equivalent to

$$
m \leq\left\lfloor\frac{p}{t_{l}}\right\rfloor
$$

In addition, the time required for robot $R_{j}$ to transport $n_{j}$ loads must be less than $T$, so we can write this condition as $p \cdot n_{j}+(j-1) \cdot t_{l} \leq T$.

As $n_{j}^{\max }$ is an integer, we have

$$
n_{j}^{\max }=\left\lfloor\frac{T-t_{l} \cdot(j-1)}{p}\right\rfloor
$$

- Case 2: As $m$ is an integer, the case $\frac{p}{t_{l}}<m<\frac{p}{t_{l}}+1$ is only possible if $\frac{p}{t_{l}}$ is not an integer. It follows that the condition is equivalent to $m=m_{0}$ with $m_{0}=\left\lceil\frac{p}{t_{l}}\right\rceil$. The time required by $R_{j}$ to transport $n_{j}$ loads must be less than $T$, i.e.

$$
n_{j} \cdot p+(j-1) \cdot t_{l}+\left(n_{j}-1\right)\left(m_{0} \cdot t_{l}-p\right) \leq T .
$$

It follows that

$$
n_{j}^{\max }=\left\lfloor\frac{T-t_{l}(j-1)-p+m_{0} \cdot t_{l}}{m_{0} \cdot t_{l}}\right\rfloor
$$

Then, we get the expression of $N_{\max }(m)=\sum_{j=1}^{m} n_{j}^{\max }$, the maximum number of loads transported by $m$ robots :

$N_{\text {max }}(m)= \begin{cases}\sum_{j=1}^{m}\left\lfloor\frac{T-t_{l}(j-1)}{p}\right\rfloor & \text { if } m<m_{0} \\ \sum_{j=1}^{m_{0}}\left\lfloor\frac{T-t_{l}(j-1)-p+m_{0} t_{l}}{m_{0} \cdot t_{l}}\right\rfloor & \text { if } m \geq m_{0}\end{cases}$

We conclude that the optimal number of robots is :

$$
m^{*}=\min \left[m \in\left\{1, . ., m_{0}\right\}: N_{\max }(m) \geq n\right]
$$

Robots load balancing In this section, we use the found minimum number of robots $m^{*}$ and the balanced distribution of tasks presented previously (Theorem 1) which always remains valid for this case.

Let $n_{j}$ be the number of loads transported by robot $R_{j}$ during the interval $[0, T]$. We have

$$
\begin{gathered}
n_{j}=\left\{\begin{array}{cl}
\left\lceil\frac{n}{m^{*}}\right] \text { if } & j \in\left\{1, \cdots, m^{*}-s\right\} \\
\left\lfloor\frac{n}{m^{*}}\right\rfloor \text { if } & j \in\left\{m^{*}-s+1, \cdots, m^{*}\right\}
\end{array}\right. \\
\text { et } \quad s=\left\lceil\frac{n}{m^{*}}\right\rceil m^{*}-n
\end{gathered}
$$

We can express the end date of the last transport cycle $C_{j}$ for any robot $R_{j}$ :

- Case 1:

The operating time of $R_{j}$ includes the transport time of $n_{j}$ loads as well as the waiting time for the first loading (see Fig. (4)). 
It follows that

$$
C_{j}=n_{j} \cdot p+(j-1) \cdot t_{l}
$$

\section{- Case 2:}

The operating time of $R_{j}$ contains the transport time of $n_{j}$ loads, the waiting time for the first loading (except the first robot $R_{1}$ ) which is proportional to the loading time $t_{l}$ and the waiting time for any other loading which is proportional to the quantity of loads $\left(m^{*} \cdot t_{l}-p\right)$ (see Fig. 5). Hence, we have

$$
C_{j}=n_{j} \cdot p+(j-1) \cdot t_{l}+\left(n_{j}-1\right)\left(m^{*} \cdot t_{l}-p\right)
$$

In the end, we can summarize our results in the following theorem.

Theorem 2. When considering a single pickup station, the optimal number of robots is

$$
m^{*}=\min \left[m \in\left\{1, \cdots, m_{0}\right\}: N_{\max }(m) \geq n\right]
$$

with $m_{0}=\left\lceil\frac{p}{t_{l}}\right\rceil$ and

$N_{\max }(m)= \begin{cases}\sum_{j=1}^{m}\left\lfloor\frac{T-t_{l}(j-1)}{p}\right\rfloor & \text { if } m<m_{0} \\ \sum_{j=1}^{m_{0}}\left\lfloor\frac{T-t_{l}(j-1)-p+m_{0} t_{l}}{m_{0} t_{l}}\right\rfloor & \text { if } m \geq m_{0}\end{cases}$

The most balanced solution has a makespan

$$
C_{\max }=\max _{1 \leq j \leq m^{*}}\left(C_{j}\right)
$$

where

$$
C_{j}= \begin{cases}n_{j} p+(j-1) t_{l} & \text { if } m^{*}<m_{0} \\ n_{j} p+(j-1) t_{l}+\left(n_{j}-1\right)\left(m^{*} t_{l}-p\right) & \text { if } m^{*} \geq m_{0}\end{cases}
$$

When applying Theorem 2 to the case with zero loading time $\left(t_{l}=0\right)$, we re-obtain Theorem 1 as the condition $\left(p \geq m \cdot t_{l}\right)$ is always true.

\section{TRANSPORT OF HETEROGENEOUS LOADS}

In this section, we extend our results to heterogeneous loads in the case of many stations. Fig. 6 presents an example with two types of loads.

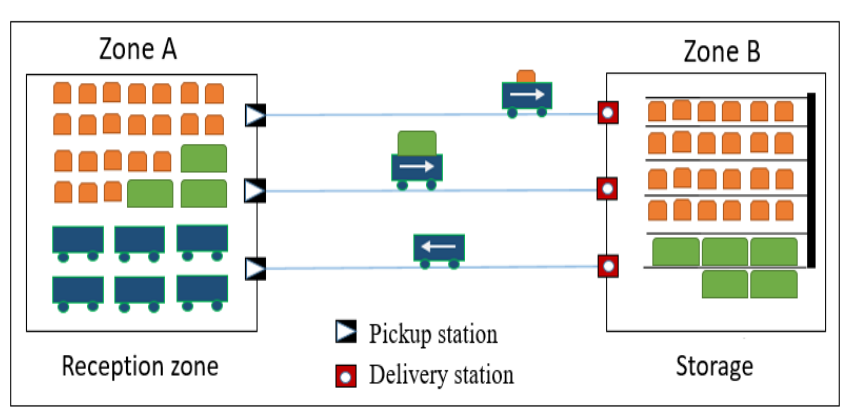

Fig. 6. A transport model with two types of loads

We assume that the weight of the load impacts the speed of a loaded robot. Without this assumption the problem remains the same as the one of section 2.2 since we use the same type of robots.

We will use the following notations:

$-n$ : total number of loads (denoted $1, \cdots, n$ )

$-v_{l i}$ : travel speed of a robot carrying load $i$

The cycle time of the load $i$ is

$$
p_{i}=d_{A B} \cdot\left(\frac{1}{v_{l i}}+\frac{1}{v_{e}}\right)+t_{l}+t_{u}
$$

We assume that $p_{i} \leq T$ for all $i$, otherwise there is no solution to the problem.

This problem can be formulated as a bin packing problem. We can consider that we have $n$ objects (loads). Each object $i$ has a size $p_{i}$. The problem is to put all the objects in bins (robots) of size $T$ (time limit). The smallest number of bins is the optimal number of robots $m^{*}$.

This problem can be formulated as an integer linear programming. Denote by $x_{i j}$ the binary variable equal to 1 if load $i$ is transported by robot $R_{j}, 0$ otherwise. Denote also by $y_{j}$ the binary variable equal to 1 if bin $j$ is used, 0 otherwise.

As there are at most $n$ needed robots, we therefore seek to minimize the number of bins used

$$
\min \sum_{j=1}^{n} y_{j}
$$

under the following constraints:

$$
\begin{array}{lr}
\sum_{i=1}^{n} p_{i} \cdot x_{i j} \leq T \cdot y_{j} & j=1, \cdots, n \\
\sum_{j=1}^{n} x_{i j}=1 & i=1, \cdots, n \\
y_{j} \in\{0,1\} & j=1, \cdots, n \\
x_{i j} \in\{0,1\} & i, j=1, \cdots, n
\end{array}
$$

There are different methods to solve this problem (see for example Toth and Martello (1990)). 
Continuous approximation If we allow a non-integer number of robots and loads, we can simplify the solution of our problem to

$$
m^{*}=\sum_{i=1}^{n} p_{i} / T
$$

\section{CONCLUSION}

This paper considers a fleet-sizing problem of robots in a logistics warehouse. An analytical approach allowed us to deal with this problem for a simple model of transporting homogeneous loads between two storage areas in the warehouse, taking into account the number of pickup and delivery stations in the system. For the case with many pickup stations $\left(n_{A} \geq m^{*}\right.$ and $\left.n_{B} \geq n_{A}\right)$, the size of the fleet is given by a simple analytical expression (see Theorem 1). For the case with a single pickup station $\left(n_{A}=1\right.$ and $\left.n_{B} \geq n_{A}\right)$, the problem is solved using a simple algorithm (see Theorem 2). We also show how to formulate the problem with heterogeneous loads and many stations by a bin packing problem and an integer linear programming.

It would be interesting to extend our model to the case with heterogeneous robots in capacity or speed.

\section{REFERENCES}

Chawla, V., Chanda, A., and Angra, S. (2018b). Automatic guided vehicles fleet size optimization for flexible manufacturing system by grey wolf optimization algorithm. Management Science Letters, 8(2), 79-90.

Chawla, V., Chanda, A., and Angra, S. (2019). Material handling robots fleet size optimization by a heuristic. Journal of Project Management, 4, 177-188.

Choobineh, F.F., Asef-Vaziri, A., and Huang, X. (2012). Fleet sizing of automated guided vehicles: a linear programming approach based on closed queuing networks. International Journal of Production Research, 50(12), 3222-3235.

Egbelu, P.J. (1987). The use of non-simulation approaches in estimating vehicle requirements in an automated guided vehicle based transport system. Material flow, 4(1), 17-32.

Ganesharajah, T., Hall, N.G., and Sriskandarajah, C. (1998). Design and operational issues in agv-served manufacturing systems. Annals of Operations Research, $76,109-154$.

Mouad, M. (2014). Architecture de COntrôle/COmmande dédiée aux systèmes Distribués Autonomes $\left(A C O^{2} D A\right)$ : application à une plate-forme multi-véhicules. Ph.D. thesis, Clermont-Ferrand 2.

Sinriech, D., Tanchoco, J., and (1992). An economic model for determining agv fleet size. International Journal of Production Research, 30(6), 1255-1268.

Toth, P. and Martello, S. (1990). Knapsack problems: Algorithms and computer implementations. Chapter 8: Bin-packing problem, 221-245.

Vivaldini, K., Rocha, L.F., Martarelli, N.J., Becker, M. and Moreira, A.P. (2016). Integrated tasks assignment and routing for the estimation of the optimal number of agvs. The International Journal of Advanced Manufacturing Technology, 82(1-4), 719-736. 